\title{
Design of Binary Band Pass Filter
}

\author{
Prof. Abhijit G. Kalbande \\ Department of Electronics \& Telecommunication Engg, P.R.M.C.E.A.M, Bandera, Maharashtra, India
}

\begin{abstract}
This paper deals with band pass filter which is located between up converter and modulator of uplink model for $\mathrm{C}$ band small satellite communication system that work digitally. The main theme of designing a digital FIR filter is to provide the better settlement solution, to improve an efficiency of the desired signal of the system and to allow adjustment of the compromise between the over shoot reduction and transition region width for practical application of the small satellite uplink system. Digital filter can be applied in speech processing applications, such as speech filtering, speech enhancement, noise reduction and automatic speech recognition among others. In this paper MATLAB Program is used to implement a band-pass FIR filter using adjustable window function based on blackmann window. The realization structure of this filter with a specific and symmetric filter coefficient is analysed and the symmetric coefficients of the filter structure are that this filter is stable, it is also linear and it has a constant group delay. And then the magnitude response and phase response of this filter are analysed and the simulation results are also described using FDA tool that is one of the Computer Aided Design tool available with MATLAB which enables design of the digital filter blocks faster and more accurate.
\end{abstract}

Keywords: uplink model, small satellite, C-band, FIR equiripple band pass digital filter, MATLAB.

\section{INTRODUCTION}

Small satellites are becoming increasingly popular due to their low cost, minimized volume, reduced design and advance time and then digital filters are the essential part of satellite communications, modern wireless communications and electronic system because it can eliminate the harmful constituent from signal.[9] Small satellite's dimensions and mass does not differ from large ones, including practically the same functions. Classical satellites are large, expensive and process of their building lasts for many years and therefore small satellites are playing a very important role in the field of remote sensing, navigation and surveillance and it necessities the use of commercial off the shelf (COTS) elements so they can be used in a lot of applications such as earth observation, education, military applications, distance learning, telemedicine, universal access, disaster recovery and television transmission in many tropical regions[5]. The frequency band used in this system is $\mathrm{C}$ band because they are mainly used for numerous Asian countries. Digital Signal Processing (DSP) is one of the fields where developments are taking place at faster rate. The DSP applications demand high speed and low power digital filters. In order to meet these requirements, the order of the digital filter must be kept as small as possible. There are various sophisticated Computer Aided Design tools are available to make the digital filter fast and power efficient. Filter design and analysis tool (FDA) is one of the Computer Aided Design tool available with MATLAB which enables design of the digital filter blocks faster and more accurate.

\section{RELATED WORK}

\subsection{C-Band Small Satellite Uplink Model:}

There are many types of band services in communication. Among them, $\mathrm{C}$ band services are essentially important for developing countries as the supporting element is inexpensive and the signal cover large area. Such services are well improved to provide data services and internet connectivity. They facilitate global communications and deliver a wide range of services in developing countries. $\mathrm{C}$ band is most popular because of less propagation problem. Rain attenuation and sky noise exists low for $\mathrm{C}$ band that resists snow effects and might have a maximum output power. The standard $\mathrm{C}$ band frequency for uplink model is 5.925 to $6.425 \mathrm{GHz}$ and that of the downlink model is 3.7 to 4.2 $\mathrm{GHz}[5]$.

The system description using in this paper is described in Fig.1. It has five main portions such as Encoder, IF Modulator, Band pass filter, Up-converter and High Power Amplifier. The input baseband signal comes into in the encoder system and passes through the modulator trail. The output of the modulator is the input of the band pass filter. The output of this filter is entered to the next stage, up-converter. And then the desired signal is caught to the last stage, high power amplifier. This stage passes on the desired input baseband signal to the antenna system. Finally, the antenna transmits the wanted signal to the transponder system through the space. In the small satellite uplink system, digital band pass filter is utilized to remove unwanted signal because the preferred baseband signal contains the unwanted factor called interference or noise. So, such noise or interference is prohibited by using digital band pass filter. The 
advantages of digital band pass filter (BPF) are that it is convenient in designing small in size and low in insertion loss. So, it is commonly used in satellite communication services [6].

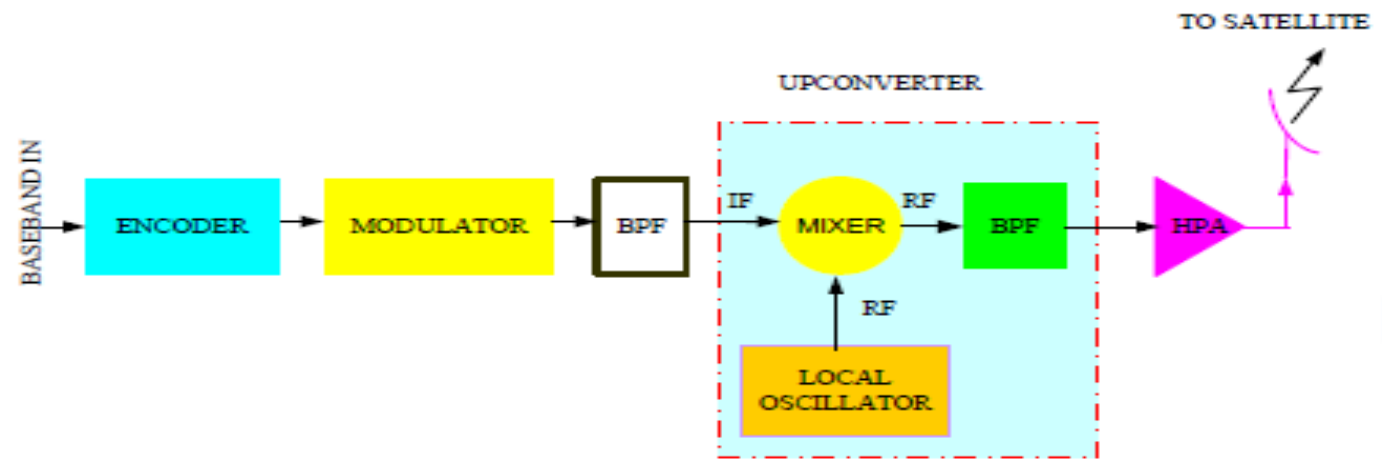

Fig.1. Block Diagram of C-Band Small Satellite Uplink System

Digital filter are two types:-

1) IIR filter(Infinite impulse response)

2) FIR Filter(Finite Impulse Response)

\subsection{IIR Filter:-}

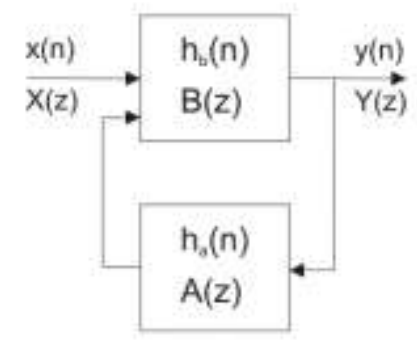

Fig.2 IIR filter block diagram

The infinite impulse response (IIR) filter is recursive structure, and it has a feedback loop. The precision of amplitude frequency characteristic is very high, and IIR filters are not linear phase.[11]

\subsection{FIR Filter:-}

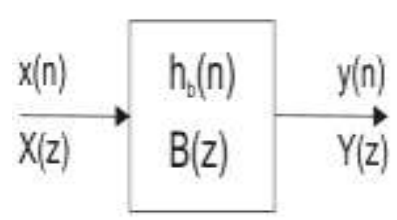

Fig.3 FIR filter block diagram

The finite impulse response (FIR) filter is one of the most basic elements in a digital signal processing system, and it can guarantee a strict linear phase frequency characteristic with any kind of amplitude frequency characteristic. Besides, the unit impulse response is finite; therefore, FIR filters are stable system. The FIR filter has a broad application in many fields, such as telecommunication, image processing, and so on.

The system function of FIR filter is,

$$
\mathrm{H}(\mathrm{z})=\sum_{\mathrm{n}=0}^{\mathrm{L}-1} \mathrm{~h}[\mathrm{n}] \mathrm{z}-\mathrm{n}
$$

Where $\mathrm{L}$ is the length of the filter, and $\mathrm{h}[\mathrm{n}]$ is the impulse response.

\subsection{FIR filter Design Techniques}

FIR filter design essentially consists of two parts:[8] 
1) Approximation problem

2) Realization problem

The approximation stage takes the specification and gives transfer function.

Realization part deals with choosing the structure to implement the transfer function which may be in the form of circuit diagram or in the form of a program. There are various methods to design FIR filter as follows:

1) Window technique.

2) Optimal filter design methods.

3) Frequency sampling method

Among them, windowing design technique is simple and convenient. Some of the windows commonly used are Blackman, Blackman-harris, Kaiser, Bohman, Chebyshew, Flat top, Gaussian, Hamming, Hann, Parzen, Rectangular, etc. So, we have designed the FIR bandpass filter using blackman window technique using FDA tool in MATLAB. The magnitude response of the bandpass filter is as shown below:

\section{SIMULATION RESULTS}

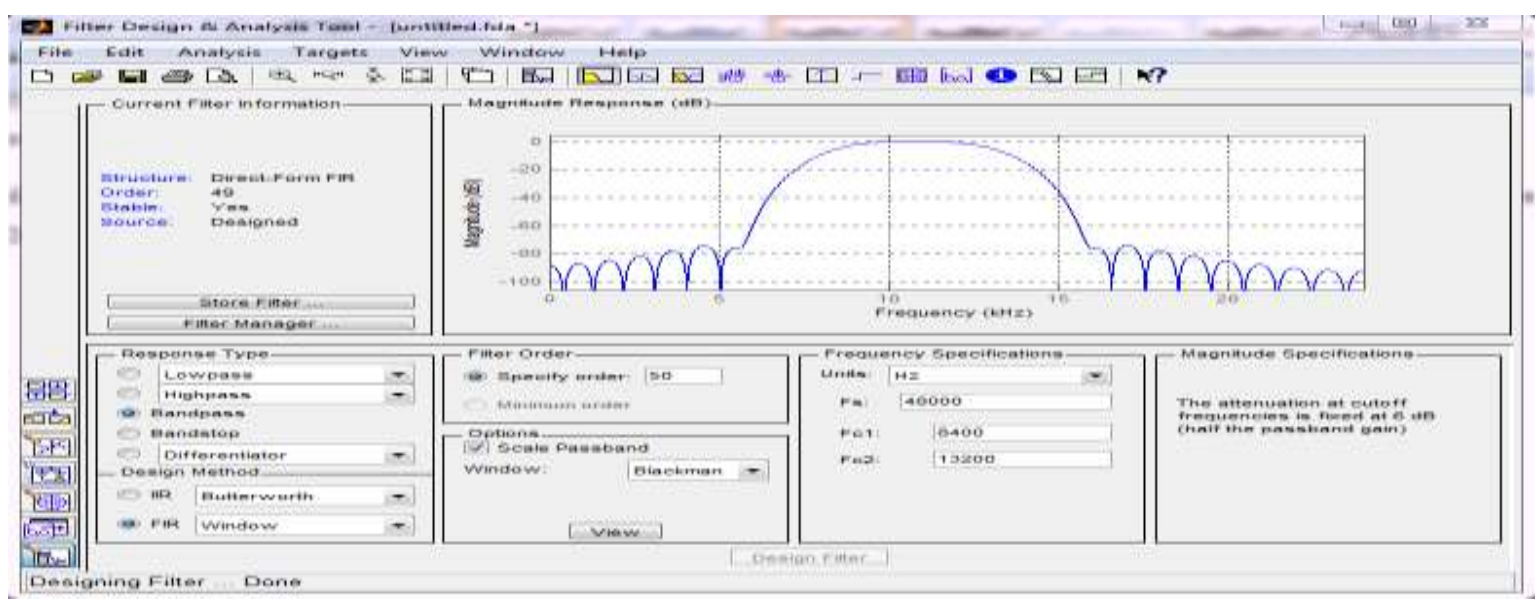

Fig.4: Magnitude response of bandpass filter using blackman window technique

In the simulation results, we got the fir bandpass filter using blackmann window technique in matlab code. This matlab code generation file is generally saved with extension such as .m, .hdl, .mdl, etc. The simulation result is shown below:

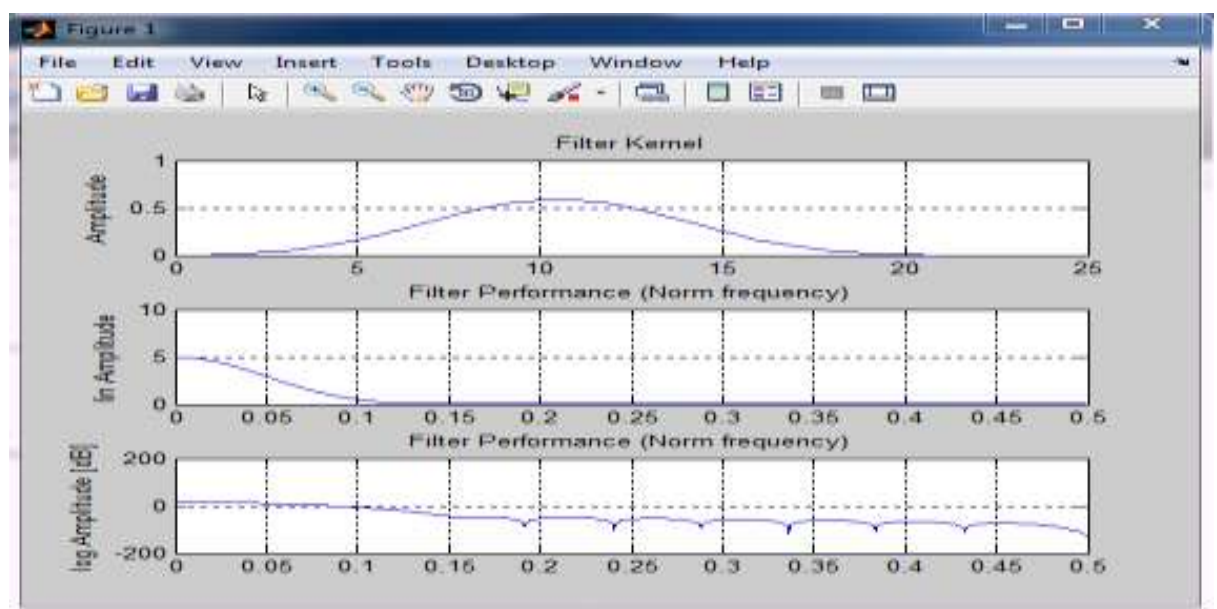

Fig.5: simulation results of the FIR filter using window technique

After dragging the FIR filter generation code by window method in Xilinx (version vivado 2014.2), we get the actual schematic of the FIR filter and the important parameters like speed, are and the power required for the desired functioning of the FIR filter so that on the basis of above parameters, we can conclude that the designed filter is more efficient and has a high of speed of operation.

\subsection{Performance Parameter}

Following are the parameters of Digital FIR Filter. 
1. Propagation Delay: Time required for a signal to pass through a given complete operating circuit, it is generally in nanosecond and is of extreme importance in simulation circuits. It is defined as the output response time with input event time. ( $\mathrm{t} 2$ - $\mathrm{t} 1)$.

2. Dynamic Power Dissipation : It is defined as the leakage power / dissipate power when CMOS circuit charging or discharging various load capacitance, also drain and some sources whenever they are switched.

$(\mathrm{P}=$ mean $(\mathrm{I}) * \mathrm{v})$. Mean $(\mathrm{I})$ - Average of current values, $\mathrm{V}$ - supply voltage.

3. Technology and Max. Power supply.

4. Area / Size : Area / Size depend on the aspect ratio of each and every module. Aspect ratio defined as, the ratio of width and length of COMS transistor which is used in the design of each and every module.

i.e. Aspect ratio $=(\mathrm{W} / \mathrm{L}) \mu \mathrm{m}$, which is separately defined for NMOS and PMOS transistor by manually.

\subsection{Result after compilation on Quartus-II:-}

The result after compilation is as given in the following table:-

Table No.1: Result after compilation on QUARTAS-II

\begin{tabular}{|c|c|c|}
\hline Specification & Filter (Direct Form) & Improved digital filter by window technique \\
\hline Entity Name & Test_df_filterI & SignalFromLookUp \\
\hline Total logic elements & $508 / 5980(8 \%)$ & $502 / 5980(8 \%)$ \\
\hline Total Pins & $51 / 185(28 \%)$ & $20 / 185(11 \%)$ \\
\hline Total thermal power dissipation & $133.81 \mathrm{~mW}$ & $104.44 \mathrm{~W}$ \\
\hline
\end{tabular}

The Altera quartus-II software is design software which provides a complete multiplatform design environment that easily adapts to your specific design needs. The quartus-II software includes solutions for all phases of FPGA \& CPLD design.[3] The Quartus-II software includes a modular compiler. The Altera FIR compiler provides a fully integrated finite impulse response (FIR) filter development environment optimized for use with Altera FPGA devices. [4]

\section{ADVANTAGES, DISADVANTAGES AND APPLICATION}

\subsection{Advantages of using FIR filter design technique}

$>$ FIR filters can have exactly linear phase.

$>$ FIR filters are automatically stable.

$>$ There are several very flexible methods for designing FIR digital filters.

$>$ FIR filters are convenient to implement.

\subsection{Disadvantages or limitations}

$>$ Linear-phase FIR filters can have long delay between input and output.

$>$ If the phase need not be linear, then IIR filters can be much more efficient to implement.

\subsection{Applications}

4.3.1. Noise suppression

$>$ Imaging devices (medical, etc)

$>$ Biosignals (heart, brain)

$>$ Signals stored on analog media (tapes)

4.3.2. Enhancement of selected frequency ranges

$>$ Equalizers for audio systems (increasing the bass)

4.3.3. Removal or attenuation of selected frequencies

$>$ Removing the DC component of a signal

$>$ Removing interferences at a specific frequency, for example those caused by power supplies.

\section{CONCLUSION}

The design of FIR filters for this uplink model is developed in this project. This filter design method is suited for electronic communication applications and the best result is acquired by using it. Finally by using this technique we can minimize the area, the low power requirements and time requirement is also reduced by providing a constant group delay. 


\section{REFERENCES}

[1] Magdy T. Hanna, - Design of Linear Phase FIR Filters with Maximally Flat Pass band II , IEEE Trans. Circuits Syst. II, 1996.

[2] Keshab.K.Parhi, VLSI Digital Signal Processing Systems Design and Implementation. A Wiley-Interscience Publication,1999

[3] J.G. Proakis, D.G. Manolakis,"Digital Signal processing Principles", Algorithms and Applications New Delhi: Prentice-Hall, 2000.

[4] Arojitroychaudhary, || FIR filter design tech. \| M.Tech. credit seminar report,Electronicn Systems Group, EE Dept, IIT Bombay, submitted November 2002

[5] F. Bernelli-Zazzera, A. ErcoliFinzi, M. Molina, and M. Cattaneo, - In-orbit technology validation for a university microsatellite II , inProc. 4th IAA Symp. Small Satell. Earth Observ., Berlin, Germany, 2003

[6] T. Vladimirova and M. Sweeting, -System-on-a-chip development for small satellite onboard data handling \| , J. Aerosp.Comput., Inform. Commun., vol. 1, no. 1, pp. 36 - 43, 2004

[7] Shanthala S, S.Y.Kulkarni-High Speed and Low Power FPGA Implementation of FIR Filter for DSP Applications, -European Journal of Scientific Research, ISSN 1450-216X Vol.31 No.1 (2009), pp. 19-28.

[8] Prof. GopalS.Gawande, - Performance analysis of FIR Digital Filter Design Techniques || , International Journal of Computing and Corporate Research, Vol. 2, Issue 1 January 2012.

[9] T. Pratt, C. Bostian ,J.Allnutt, "Satellite Communication \|| John Wiley and Sons, 2nd Edition

[10] Department of Electronics \& Communication Engineering Jaypee Institute of Information Technology Sec-62,Noida, India

[11] Design and analysis of FIR filters based on matlab, author: Su Peng,subject: Electrical Engg,Linnaeos University,school of computer science,physics \& maths 